Revista Tecné, Episteme y Didaxis: TED. Año 2014, Número Extraordinario. ISSN Impreso: 0121-3814, ISSN web: 2323-0126

Memorias, Sexto Congreso Internacional sobre Formación de Profesores de Ciencias. 08 al 10 de octubre de 2014, Bogotá

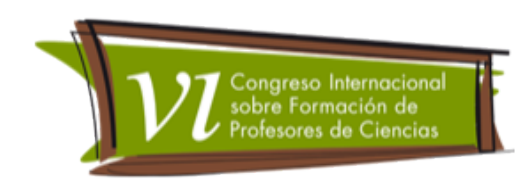

\title{
Consideraciones teóricas sobre la formación de profesores de ciencias en la interfaz universidad-escuela: una investigación internacional en desarrollo
}

Martínez P. Leonardo F1, Parga, L. Diana L2, Pinzón N. Yadira A33, Salazar M. Viviana4.

Categoría 2. Trabajos de investigación (en proceso o concluidos).

\section{Resumen}

En esta ponencia se presentan los principales elementos teóricos y algunos resultados de un programa de formación de profesores de ciencias en la interfaz universidadescuela, el cual es desarrollado en Colombia y Brasil. Dicho programa tiene como objetivo analizar los alcances y límites de la conformación de pequeños grupos de investigación en la escuela y a partir de dicho análisis establecer recomendaciones curriculares para la Enseñanza de las Ciencias centrada en el la discusión de cuestiones sociocientíficas y ambientales. Los resultados parciales evidencian la conformación de pequeños grupos de investigación en Colombia y el inicio de un diálogo interesante con pequeños grupos de investigación de Brasil.

\section{Palabras clave}

Investigación en la escuela, cuestiones sociocientíficas y ambientales, currículos alternativos para la formación de profesores.

\section{Introducción}

El proyecto internacional de investigación denominado Programa de Formación de Profesores de Ciencias en la interfaz universidad-escuela (U-E) adelantando interinstitucionalmente entre la Universidade Estadual Paulista de Brasil (UNESP) y la Universidad Pedagógica Nacional (UPN) de Colombia es financiado parcialmente por CAPES y COLCIENCIAS a partir del contrato 531-2012 y ha cumplido 12 meses de desarrollo. En este periodo se han adelantado talleres, encuentros y foros de formación

\footnotetext{
1 Profesor Departamento de Química, Universidad Pedagógica Nacional, Bogotá- Colombia. lemartinez@pedagogica.edu.co

2 Profesora Departamento de Química, Universidad Pedagógica Nacional, Bogotá- Colombia. dparga@pedagogica.edu.co

3 Profesora institución privada de Educación Media, Bogotá-Colombia. Magíster en Docencia de la Química. yapin_15@hotmail.com

4 Profesora de la Secretaria de Educación de Bogotá-Colombia. hadadeloslagos@yahoo.com
} 
Revista Tecné, Episteme y Didaxis: TED. Año 2014, Número Extraordinario. ISSN Impreso: 0121-3814, ISSN web: 2323-0126

Memorias, Sexto Congreso Internacional sobre Formación de Profesores de Ciencias. 08 al 10 de octubre de 2014, Bogotá

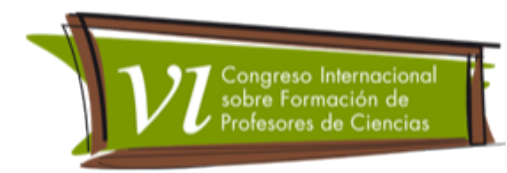

con profesores en ejercicio y en formación inicial, en aras de construir pequeños grupos de investigación (PGI) centrados en el análisis del tratamiento de cuestiones sociocientíficas (CSC) y ambientales. La conformación de estos grupos se ha fundamentado en la formación crítica del profesor y en la interdisciplinariedad en términos de posibilitar el desarrollo de experiencias docentes significativas para los distintos profesores de la escuela. Los primeros avances del proyecto llaman la atención para la transformación y renovación de la Enseñanza de las Ciencias a partir del diálogo U-E para articular investigaciones escolares e investigaciones didácticas, en aras de favorecer la formación ciudadana de estudiantes de educación básica y media en cuestiones controvertidas de ciencia y tecnología (C\&T).

En un primer momento, presentamos elementos teóricos sobre el diálogo U-E y los presupuestos curriculares que caracterizan los contenidos, metodologías, formas de evaluar y compromisos político-sociales del programa de formación. A partir de esta fundamentación se analizan algunas consideraciones de los profesores involucrados en el programa evidenciando que constituye una propuesta abierta y flexible que no está determinada por expertos $u$ objetivos prescritos. Así se constituye una propuesta curricular abierta en contenidos e intencionalidades construidas con los participantes a propósito de los proyectos que cada PGl perfila desarrollar.

El proyecto de investigación marco de este trabajo propone una metodología de investigación colaborativa entre los profesores de los PGI y los profesores universitarios, fundamentada en posibilitar la constitución de asociaciones libres, en la que estos agentes puedan tener la oportunidad de reflexionar sobre su formación permanente, junto a sus pares de la escuela y la universidad. Además de eso, la autonomía de estos profesores debe ser una búsqueda constante, como un cuestionamiento de los currículos y políticas educativas, en el sentido expuesto por Contreras (2002). Así, la autonomía vista sobre la perspectiva de la teoría crítica, tiene en la intersubjetividad el medio para la formación social y para la emancipación.

Frente a estos preceptos, el trabajo propuesto se enmarca como una investigación cualitativa colaborativa coherente con los planteamientos de Kemmis e McTaggart (2003) ese tipo de investigación no se reduce al objeto de estudio, ni a los métodos o técnicas utilizadas, pero si a las acciones y comprensiones de las acciones de los sujetos involucrados en el proceso educativo. 
Revista Tecné, Episteme y Didaxis: TED. Año 2014, Número Extraordinario. ISSN Impreso: 0121-3814, ISSN web: 2323-0126

Memorias, Sexto Congreso Internacional sobre Formación de Profesores de Ciencias. 08 al 10 de octubre de 2014, Bogotá

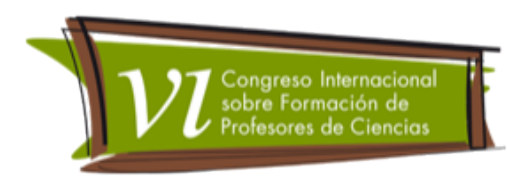

\section{Principios teóricos del programa de formación de profesores en la interfaz U-E y aspectos curriculares}

La relación entre la U-E ha estado marcada por la indefinición e imprecisión en los fines y proyectos comunes, lo cual ha generado una frágil vinculación y distanciamiento progresivo entre ambas, por tal motivo es indispensable repensar los objetivos y propósitos de interés mutuo como lo plantean Rodríguez y Garzón (2003) desde un contexto democrático que considere esta correlación como la articulación que pueden lograr estas dos instancias desde sus respectivas misiones educativas.

La escuela de la sociedad actual se presenta como un espacio en donde confluyen tensiones y conflictos, lo que implica redefinir su función, crear puntos de relación entre lo pedagógico y el accionar comunitario, convirtiéndose en un espacio de participación y compromiso genuino, donde los distintos actores intervengan de forma activa, voluntaria y equitativa en los asuntos que les interesan y preocupan, de esta forma es posible fomentar la cultura de negociación, generar acuerdos centrados en actividades de investigación, proponer esquemas de organización en espacios enmarcados por el trabajo en equipo y el aprendizaje cooperativo.

De allí, la pertinencia de una teoría de la acción comunicativa en el contexto formativo que permita fundar las bases para una teoría crítica, social, progresista y emancipadora, desde la perspectiva del diálogo y el consenso (Habermas, 1987) entre la universidad y la escuela, posibilitando una comunicación acordada, donde cada participante de la comunidad educativa pueda expresar sus propias interpretaciones y someter sus ideas subjetivas, objetivas y sociales a consenso, con el fin de promover conciencia a través de reflexiones sobre sí mismos y sobre sus actuaciones.

Coherentemente con los aspectos teóricos expuestos anteriormente, el currículo del programa de formación en la interfaz U-E hace alusión a lo disciplinar, a lo metadisciplinar, a lo pedagógico y a lo contextual.

En cuanto a lo disciplinar el profesorado debe tener una formación científica que le permita, primero a él, tener juicios sobre la conveniencia o no de CSC, tales como los transgénicos, o del uso de combustibles fósiles o la producción de nuevos materiales, por dar algunos ejemplos, es decir, que hoy conocer la disciplina a enseñar es más que saber los conceptos, principios, teorías, modelos, leyes, sus experimentos, que son fundamentales para entender lo que ocurre a nuestro alrededor, sino que además significa conocer sobre los temas controvertidos que hoy tiene C\&T, dado que son asuntos en los que todos los ciudadanos estamos involucrados porque de alguna manera nos afectan. 
Revista Tecné, Episteme y Didaxis: TED. Año 2014, Número Extraordinario. ISSN Impreso: 0121-3814, ISSN web: 2323-0126

Memorias, Sexto Congreso Internacional sobre Formación de Profesores de Ciencias. 08 al 10 de octubre de 2014, Bogotá

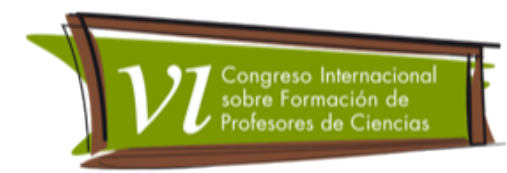

En cuanto a lo pedagógico, didáctico y contextual la formación permanente del profesorado debe orientarse hacia la indagación en la práctica, pues se requiere un profesor reflexivo, crítico, e investigador de su proceso de educación/enseñanzaaprendizaje que contemple el individuo, el aula, el entorno, el colectivo, la institución, la comunidad y sus actitudes en cada uno de estos contextos. Además, dicha formación debe propender por los diseños curriculares del propio profesorado en conformidad con un trabajo colaborativo y contextualizado.

\section{Avances del programa de formación de profesores de acuerdo con sus actores}

Inicialmente el programa de formación encontró que los docentes en las escuelas consideran que las investigaciones educativas realizadas en las universidades presentan un mínimo aporte en torno a la transformación de la escuela y que la investigación que se realiza desde la escuela con el ánimo de innovar, presenta una carga ideológica, un lenguaje y unas lógicas diferentes a las determinadas por los expertos de la universidad, como se muestra en el siguiente apartado donde PP hace referencia a un profesor participante del PGI:

PP: Es importante que se tenga en cuenta lo que los profesores realizamos en los colegios en torno a la investigación enfáticamente sobre los problemas de aprendizaje, pues en la mayoría de ocasiones las facultades de educación en su afán por innovar traen a las escuelas muchas propuestas que terminan fracasando, así como se evidencia por ejemplo en los practicantes que no tienen herramientas consolidadas para afrontar la realidad escolar ${ }^{5}$

De allí la importancia de abordar la desarticulación entre la formación del profesor de ciencias y la realidad de la escuela, analizando la interacción y compromiso de la universidad y la escuela a través de la conformación de PGl, es importante para que cada participante se asuma como sujeto activo en situaciones de cambio y transformación, que permitan establecer recomendaciones curriculares y criterios de formación hacia el abordaje de perspectivas y problemáticas contextuales del siglo $X X I$, tales como las CSC y ambientales.

En la conformación de los PGI se han encontrado factores positivos encaminados a la aproximación de la universidad con la escuela a través de la interdisciplinariedad, posibilitando la reflexión de la labor docente y oportunidades de intercambio de experiencias a nivel internacional.

5 Fragmento tomado de la relatoría relación U-E, la cual se realizó en la visita de presentación del Programa. 
Revista Tecné, Episteme y Didaxis: TED. Año 2014, Número Extraordinario. ISSN Impreso: 0121-3814, ISSN web: 2323-0126

Memorias, Sexto Congreso Internacional sobre Formación de Profesores de Ciencias. 08 al 10 de octubre de 2014, Bogotá

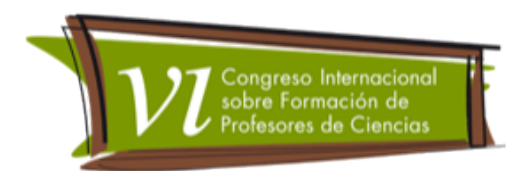

De esta manera, se han visualizado algunas limitaciones relacionadas con los tiempos de encuentro dentro de las instituciones educativas, debido a que los profesores vinculados a los PGI tienen horarios diferentes, es complejo encontrar tiempos comunes, por lo tanto comentan que el programa exige tiempo extra que es difícil acordar: "PP: No hay tiempo extra clase para estudiar y puesto que decidí tener dos hijos y están muy pequeños (...). En esta etapa de la vida me gusta dedicarles mi tiempo"6. Además, aunque se hace énfasis en la interdisciplinariedad que promueve el programa de formación para enriquecer la interfaz U-E, algunos de los docentes consideran necesaria una capacitación en ciencias antes de ingresar al PGI, para entender los diferentes lenguajes del conocimiento.

En cuanto al currículo se ha evidenciado una construcción abierta y de libre asociación, ya que se pueden involucrar diferentes espacios académicos, que orienten los profesores investigadores del proyecto, pero además profesores de diferentes áreas para la conformación del pequeño grupo de investigación (PGI) en la escuela, además se relacionan profesores del doctorado de la UNESP Brasil y del doctorado interinstitucional en Educación de la UPN:

El profesor universitario 1 plantea que por ello, el proyecto se pensó desde la libre asociación y desde las experiencias de ellos (profesores participantes). Él da el ejemplo de una profesora (...) que solo había logrado articularse a trabajar con una profesora de biología (es decir que ese PGI tendría un nivel inicial de interdisciplinariedad, biología y química) (Acta 9, del 2 de mayo de 2013).

La profesora universitaria 2 informa que se han recibido por correo cuatro actas de conformación de los PGI de correspondientes a los siguientes profesores (...) profesora A, cuenta con la participación de cuatro profesores en el PGI de las áreas de Sociales, Filosofía, Idiomas y Ciencias Naturales. Para trabajar la CSC referente a la explotación minera en Colombia (Acta 14 del 12 de julio de 2013).

¿ Hasta el momento cómo caracteriza el programa de Formación de profesores en la interfaz U-E en el cual participan?, la profesora coordinadora del PGI del Colegio Distrital SaludCoop Norte establece: el propósito siempre es invitar a participar voluntariamente a los profesores, por ejemplo cuando la profesora universitaria 1 fue al colegio a realizar la presentación del proyecto, motivo muchísimo, pero dentro del grupo de profesores que no manejan muchas cosas, que si se manejan en la maestría o en la universidad, se presenta mucha confusión del proyecto(....) ya que en los

\footnotetext{
6 Fragmento tomado del cuestionario de identificación de la relación U-E aplicado a los profesores participantes del programa.
} 
Revista Tecné, Episteme y Didaxis: TED. Año 2014, Número Extraordinario. ISSN Impreso: 0121-3814, ISSN web: 2323-0126

Memorias, Sexto Congreso Internacional sobre Formación de Profesores de Ciencias. 08 al 10 de octubre de 2014, Bogotá

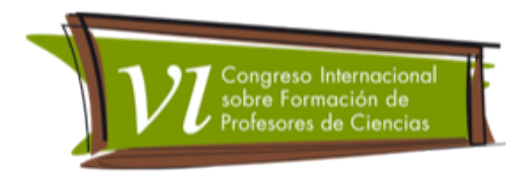

colegios no hay mucha cultura de investigación didáctica, existe la cultura de la investigación científica (método científico), basada en la metodología cuantitativa, en donde hay solo estadísticas y datos. Pero el proyecto muestra algo distinto a lo tradicional (Transcripción del taller con profesores del PGI, 9 de octubre de 2013).

El programa ha viabilizado un espacio de interacción entre los 7 PGI de Colombia y dos 2 PGI de Brasil, esto es realizado a través de foros vía skype, en los cuales se presentan avances de los proyectos y en los foros virtuales disponibles en la página del grupo Alternaciencias (www.alternaciencias.com). En estos espacios se ha discutido sobre proceso de autonomía, heteronomía y antinomia en la formación de profesores en la interfaz U-E, así mismo, se contempla el análisis de las características de las CSC y sU abordaje en las clases de ciencias de acuerdo con los objetivos contemplados en los proyectos de los PGI.

\section{Conclusiones}

Los avances teóricos y metodológicos del programa de formación de profesores de ciencias en la interfaz U-E denotan una construcción flexible y colectiva, en la cual se ha construido un compromiso reflexivo de los docentes participes de los PGI pese a las limitaciones y factores agregados que acarrea la realidad escolar, la participación activa de la universidad en las escuelas y su constante dinamización y orientación acorde con las necesidades de cada contexto escolar.

Para superar currículos de formación de profesores prescritos por facultades de educación o expertos, es necesario pensar en programas contextualizados, que definan sus contenidos, objetivos, estrategias en negociación con los intereses y necesidades del profesorado y de las instituciones mismas que reconocen sus propias problemáticas, esto para formar a un profesor investigador, reflexivo, crítico, que eduque, que enseñe la C\&T no solo desde la perspectiva disciplinar, sino desde los planteamientos de una nueva epistemología, con implicaciones éticas, morales, políticas y económicas.

\section{Referencias bibliográficas}

Contreras, J. (2002). A autonomia de professores. São Paulo: Cortez.

Habermas, J. (1987). Teoría de la acción comunicativa. Racionalidad de la acción y racionalización social. Madrid: Taurus.

Kemmis, S. y McTaggart (2003). Participatory Action Research. En: Denzin, N y Yvonna, L. Strategies of qualitative inquirí. EUA: Sage Publications. 
Revista Tecné, Episteme y Didaxis: TED. Año 2014, Número

Extraordinario. ISSN Impreso: 0121-3814, ISSN web: 2323-0126

Memorias, Sexto Congreso Internacional sobre Formación de

Profesores de Ciencias. 08 al 10 de octubre de 2014, Bogotá

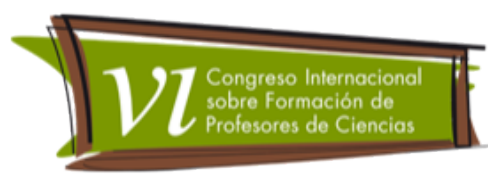

Rodríguez, J. y Garzón, J. (2003). Cooperación escuela-universidad y construcción del currículo: Programa de fortalecimiento de la capacidad científica en la educación básica y media. Universidad Nacional. 